\title{
Prostate cancer in rural communities and its effect upon African American men
}

\begin{abstract}
Prostate cancer is one of the most prevalent cancers among men in the world. However, men of African American or African descent are disproportionately affected with mortality rates twice that of white males. Factors that contribute to prostate cancer are an increase in age and genetic factors. Currently, there is research being done on the link between prostate cancer and poor diet. However, this causal link has not been made. African American men face higher rates of mortality due to prostate cancer because of a lack of education pertaining to prostate cancer, a distrust of the healthcare system and infrequent visits to their physician. These factors are then compounded by being located in a rural location where access to healthcare is scarce. With a robust educational intervention targeting this population many of these deaths can be prevented via secondary prevention i.e. prostate specific antigen (PSA) screenings and digital rectal exams (DREâ€ $€^{\mathrm{TM}_{\mathrm{S}}}$ )
\end{abstract}

Keywords: prostate cancer, rural communities, inflammation, abnormal growth, definitive factor, lymph nodes, antigen, bladder, penis
Volume 4 Issue I - 2016

Darry Coverson
Mercer University, USA

Correspondence: Darry Coverson, Mercer University, USA, Tel (404) 8380230, Email coverson_d@med.mercer.edu

Received: June 19,2015 | Published: January II, 2016
Abbreviations: PSA, prostate specific antigen; DRE, digital rectal exams; SES, separate socioeconomic status; HBM, health belief model

\section{Prostate cancer}

Prostate cancer is one of the most prevalent cancers among men in the world. However, men of African American or African descent are disproportionately affected with mortality rates twice that of white males. Factors that contribute to prostate cancer are an increase in age and genetic factors. Currently, there is research being done on the link between prostate cancer and poor diet. However, this causal link has not been made. African American men face higher rates of mortality due to prostate cancer because of a lack of education pertaining to prostate cancer, a distrust of the healthcare system and infrequent visits to their physician. Being located in a rural location where access to healthcare is scarce then compounds these factors. With a robust educational intervention targeting this population many of these deaths can be prevented via secondary prevention i.e. prostate specific antigen (PSA) screenings and digital rectal exams (DRE's).

Prostate cancer is the most prevalent cancer among men. In the United States in 2009, 206, 640 men were diagnosed with prostate cancer, and of these, $13.6 \%(28,088)$ of men died. ${ }^{1}$ Cancer is the abnormal growth of cells within the body and is named for the region or area that the cancer originates. The prostate is part of the male reproductive system and includes the following: the penis, prostate, and testicles. The prostate serves an important reproductive function that includes the making of semen. ${ }^{1}$

The prostate is located below the bladder and adjacent to the rectum; this makes the detection of prostate cancer very difficult without screenings. The prostate is the size of a walnut and surrounds the urethra; the urethra is the tube that connects the bladder to the penis and it empties the bladder's contents. The prostate increases in size as men age and with the increase in size comes the narrowing of the urethra. A narrowing urethra decreases the urine flow and prolongs the emptying of the bladder that is a symptom of prostate cancer but is not an indicator that prostate cancer is present.

Furthermore, there are several prostatic changes that occur with an increase in a man's age that aren't indicative of prostate cancer and they are as follows: passing urine more often during the day, having the sudden or urgent need to pass urine, a decrease in urine flow, a burning sensation while passing urine, and the need to pass urine several times during the night (National Institutes of Health, 2009). ${ }^{2}$ Again, these symptoms are not indicative of prostate cancer but do serve as a signal that there could be a problem in the future with a condition known as prostatitis caused by inflammation of the prostate gland (NIH, 2009).

Without routine screenings for prostate cancer and it being left untreated the cancer can spread directly into other neighboring areas of the body such as the bladder, stomach, blood stream and even implant itself in the bones of the spine. The end result of undiagnosed prostate cancer is the spread into the lymph nodes and vessels. ${ }^{3}$ Vessels are similar to veins but instead of transporting blood the lymph node vessel transports milky white fluid that contains waste from the cells. The metastasis into the lymph node vessels serve as a vehicle for the transport and spread of the disease throughout the body, eventually leading to death.

Prostate cancer has four stages of progression: stage 1, stage 2, stage 3 , and stage 4 (cite). Each stage is distinctive but may take years to progress from stage to stage. Stage 1 or T1 (tumor stage 1) is the infancy stage of the disease; the cancer is so small that it cannot be detected nor felt with the digital rectal exam. Stage 2 or T2 is when the tumor can be detected via rectal examination yet; the cancer is still 
located within the prostate. Stage 3 or T3 is indicated by the spread of the tumor to the outside of the prostate gland. This is the stage where the invasion of the cancer into neighboring areas occurs as mentioned previously. Lastly, stage 4 or T4 is the spread of the tumor and cancer into surrounding area and invasion of the tissue in areas such as the rectum, bladder and pelvic muscles. At this time there are no definitive symptoms that will directly reflect the presence of prostate cancer. However, it is recommended that if any symptoms are present that men should speak with their doctor. Again, most of the exams measure levels of PSA, or prostate specific antigens, however, high PSA levels do not provide correlations to the presence of the disease (cite). ${ }^{4}$

Prostate cancer has an inversely proportionate relationship with age (cite); this means that as the age of males increases so does the incidence of prostate cancer incidence and diagnosis. ${ }^{5}$ Men of rural locations experience a higher incidence of prostate cancer. The average prostate cancer diagnosis age from 2006-2010 was 66 years of age; when compared to rural Georgia, the average age was 63 years of age (Steenland, 2011). These findings indicate a three year time frame where these men could potentially $\mathrm{z}$ time to progress. The ageadjusted incidence rate was 152.0 per 100,000 men per year (cite). The age-adjusted death rate was 23.0 per 100,000 men per year; these rates are between 2006 and 2010 (The National Cancer Institute, 2012). (Table $1 \& 2$ ) indicates the incidence of prostate cancer by race distribution and incidence rates by race.

Table I Incidence Rates by Rac

\begin{tabular}{ll}
\hline Race/Ethnicity & \\
\hline All Races & 152.0 per 100,000 men \\
White & 144.9 per 100,000 men \\
Black & 228.5 per 100,000 men \\
Asian/Pacific Islander & 81.8 per 100,000 men \\
American Indian/Alaska Native & 77.8 per 100,000 men \\
Hispanic & 125.8 per 100,000 men \\
\hline
\end{tabular}

Based on rates from 2008-2010, 15.33\% of men born today will be diagnosed with cancer of the prostate at some point during their lifetime (cite). This number can also be expressed as a rate of 1 in 7 men being diagnosed with cancer of the prostate during their lifetime (The National Cancer Institute, 2012). These statistics are known as the lifetime risk of developing cancer. As men increase in age, so too does their probability of developing prostate cancer, making prostate cancer the most abundant form of cancer for men (cite) As of today, there are no definitive factor that has been associated with cause, however, several risk factors have been shown to play a role in association. These are increased age, family history, and race (CDC, 2013). However, one covariate found to be positively associated with the development of prostate cancer and death is race (cite). In particular, African-American men have a much higher risk of prostate cancer development than any other group of American men; the mortality rate is twice that of European-American men (cite). The lack of education and knowledge about prostate cancer is a major contributor to the increased burden of disease for African American men and for Black men in general (Kabore, 2013). Furthermore, data indicates that African American men regardless of geographic location were less likely than white men to receive treatment for prostate cancer via surgery after adjusting for income (Steenland, 2011).
Table 2 Indicates the mortality of prostate cancer by race distribution

\begin{tabular}{ll}
\hline Race/Ethnicity & \\
\hline All Races & 23.0 per 100,000 men \\
White & 21.2 per 100,000 men \\
Black & 50.9 per 100,000 men \\
Asian/Pacific Islander & 10.1 per 100,000 men \\
American Indian/Alaska Native & 20.7 per 100,000 men \\
Hispanic & 19.2 per 100,000 men \\
\hline
\end{tabular}

The current trend of prostate cancer is an increase in incidence rate, and, on the surface, may be alarming . However, upon careful examination of the data, this may not prove to be accurate. In general, people are living longer today than previous generations. With this longevity, we can expect an increase in the incidence, prevalence and overall diagnosis of diseases associated with age, including prostate cancer. This does not serve as a consolation to those who are diagnosed with this disease. Many men see the diagnosis of prostate cancer as a death sentence and may ignore the symptoms and neglect their health (cite). The determinants or associated factors for all men are the predominantly the same; however African American men and men of African descent are disproportionately affected by this disease. The determinants are even more pronounced in rural populations where access to healthcare is either nonexistent or difficult to reach due to barriers that rural population must negotiate on a daily basis. Rural communities deal with geographic barriers, technological barriers as well as others. Research indicates that there are several obstacles that factor into the increased risk, morbidity, and overall prevalence of the disease in African American men (cite). These obstacles include individual level obstacles, institutional obstacles and institutional deficits (Wray, 2009). In general the attitudes associated with prostate cancer are feeling of indifference and denial that compound the problem when speaking of a population that is less educated about prostate cancer and is therefore less likely to get prostate cancer screenings. This is further illustrated in a Georgia study that revealed poor communication with a physician is particularly prevalent among African American men in comparison to white men and these differences were associated with not choosing prostate cancer treatment in the Southwest Georgia area (Steenland, 2011).

Furthermore, there are persistent well-known incidents of unequal treatment towards African Americans involving healthcare and its access (Wray, 2009). Many reminiscent of the Tuskegee syphilis studies conducted between the 1930's and 1970's. African American men or men of African descent should be the target of a robust educational intervention with constructs from the HBM (health belief model) due to the preponderance of the disease within this population as well as the mortality rates being disproportionate to other ethnic groups such as European American men. Knowledge is power; knowledge of prostate cancer would empower African American men. An increase in knowledge would also address African American men's attitudes towards prostate cancer. Prostate cancer knowledge would also allow African American men to be less fearful when going to the doctor and engender a more collaborative relationship with their physician.

Ironically, rural populations as a whole no matter the race or gender experience more infrequent visits to physicians as well (Baldwin, Andilla, Porter, Rosenblatt, Patel \& Doescher, 2013). As previously indicated, geographic barriers and limited availability of healthcare 
professionals, in particular cancer specialists have an negative influence on early prostate cancer treatment options for rural men (Baldwin, Andilla, Porter, Rosenblatt, Patel \& Doescher 2013) . To better conceptualize these findings we can turn to other studies as well. When exploring other articles and data we see that in rural and urban Georgia, white men with prostate cancer has surgery more frequently than African American men, although the data would suggest that this may be caused more by income discrepancies than race (Steenland, 2011). However, it is impossible to separate Socioeconomic status (SES)from other morbidity and mortality measures because all of these factors certainly play a role in overall health and wellbeing [Not true, plenty of data exists that allow for this analysis including MIDUS-II).

Risk of prostate cancer among African American men is indicated by the disproportionate mortality rate, is associated with an increase in age and genetic factors, but is mediated by the absence of education relating to prostate cancer, a historical distrust of the healthcare system, and a lack of quality healthcare that is equitable to their European American male counterparts. Given that there is insufficient prostate cancer knowledge, infrequent visits to the physician and distrust of the medical system moderate the causes and that increased age and African descent exist prior to the causes.

African American men have a disproportionate rate of mortality related to prostate cancer, regardless of geographic location (cite). African American men they will experience fewer or infrequent visits to a physician, and will take a back seat to the financial or economic issues that plague African Americans in general as seen in data previously shown (cite). Many of the deaths can be prevented and mitigated with an emphasis on prostate cancer awareness and education that is tailored to African American men with the use of the health belief model (HBM). ${ }^{5}$ Health education must be tailored to the population, be culturally sensitive, and acknowledge the historical perspective of healthcare inequality that lingers within the community. With an acknowledgement of these factors, education about prostate cancer may help overcome obstacles, and deaths mitigated.

\section{Acknowledgements}

None.

\section{Conflict of interest}

The author declares no conflict of interest.

\section{References}

1. Centers for Disease Control and Prevention. 2013.

2. Fasnéwindé Aristide Kabore T K. Knowledge and Awareness of Prostate Cancer among the General Public in Burkina Faso. $J$ Cancer Educ. 2014;29(1):69-73.

3. National Institute of Health. 2009.

4. Ricardo J, Wray SM. Changing the conversation about prostate cancer among African Americans: results of formative research. Ethn Health. 2009;14(1):27-43.

5. The National Cancer Institute. 2012. 